\title{
Enhanced recovery after hepatopancreaticobiliary surgery: A single-center case control study
}

\author{
Fakhar Nasir ${ }^{1}$, Zeeshan Hyder ${ }^{1}$, Amir Kasraianfard ${ }^{1}$, Ali Sharifi ${ }^{2}$, \\ Abdolhamid Chavoshi Khamneh ${ }^{1}$, Seyed Yahya Zarghami ${ }^{1}$, and Ali Jafarian ${ }^{1}$ \\ ${ }^{1}$ Liver Transplant and Research Center, Imam Khomeni Hospital, Tehran University of Medical Sciences, Tehran, \\ ${ }^{2}$ Department of General Surgery, Hamedan University of Medical Sciences, Hamedan, Iran
}

\begin{abstract}
Backgrounds/Aims: The aim of this study was to find the safety and effectiveness of enhanced recovery after surgery (ERAS) in patients who undergo hepatopancreaticobiliary (HPB) surgeries and its association with the postoperative complications and survival rate of the patients. Methods: This study was conducted on patients who underwent HPB surgeries in Imam Khomeini Hospital Complex, Iran from 2018 to 2020. Patients who underwent surgery after from 2019 to February 2020 considered as the ERAS group $(n=47)$ in which ERAS was implemented postoperatively including removing nasogastric tube and initiating surgical diet at 6 hours post operation, and removing intraabdominal drains and Foley catheter at postoperative day one. Other patients $(n=43)$ were considered as the control group in which conventional postoperative care was implemented. Results: Ninety patients with the mean age of $47.3 \pm 13.3 \mathrm{yrs} / \mathrm{old}$ (range= 17-76) including 39 females were enrolled into the study. There were no significant differences between the demographic and preoperative comorbidities between the two groups. Pain severity of the patients in the ERAS group was significantly lower than the control group (visual analogue scales of $3.4 \pm 0.77$ vs. $4.47 \pm 0.88, p<0.001$ ). However, there were no significant differences between the other postoperative data between the two groups. One patient in each group died during hospitalization period due to myocardial infarction. Conclusions: ERAS may be safe and effective in patients who undergo HPB surgery and may be associated with less severe postoperative pain. (Ann Hepatobiliary Pancreat Surg 2021;25:97-101)
\end{abstract}

Key Words: Enhanced recovery after surgery; Hepatopancreaticobiliary surgery; Postoperative pain

\section{INTRODUCTION}

Enhanced recovery after surgery (ERAS) firstly, introduced in 1997 is substantially an evidence based approach aimed to lessen length of hospital stay by initiating oral feeding and removing nasogastric tube, drains or Foley catheter earlier in postoperative period. ${ }^{1,2}$ Since then, it was implemented in different types of surgical procedures such as colorectal, urological and esophageal cancer surgeries having promising results and very few complications compared to conventional postoperative management. ${ }^{3-5}$

Several studies were published to show the effectiveness and safety of enhanced recovery in patients undergoing hepatectomy surgeries. ${ }^{6,7}$ In 2016, the recommendations of the ERAS society were published officially regarding perioperative management in hepatopancreaticobiliary (HPB) surgeries. ${ }^{8}$ However, there has been always a fear to start oral feeding after major HPB surgeries like Whipple procedure due to a concern regarding postoperative complications including pancreatic fistula, delayed gastric emptying, intraabdominal infection. ${ }^{9}$ And so, many surgeons still believe that delaying oral feeding may protect the anastomosis in such major surgeries.

Therefore, the aim of this study was to find the safety and effectiveness of ERAS in patients who undergo HPB surgeries and its association with the postoperative complications and survival rate of the patients.

Received: May 18, 2020; Revised: August 9, 2020; Accepted: August 10, 2020

Corresponding author: Zeeshan Hyder

Liver Transplant and Research Center, Imam Khomeni Hospital, Tehran University of Medical Sciences, End of Keshavarz Blvd, Tehran 1419733141, Iran

Tel: +98-2161190, Fax: +98-2166581604, E-mail: drzeeshanhyder@gmail.com

Copyright (C) 2021 by The Korean Association of Hepato-Biliary-Pancreatic Surgery

This is an Open Access article distributed under the terms of the Creative Commons Attribution Non-Commercial License (http://creativecommons.org/ licenses/by-nc/4.0) which permits unrestricted non-commercial use, distribution, and reproduction in any medium, provided the original work is properly cited. Annals of Hepato-Biliary-Pancreatic Surgery • pISSN: 2508-5778 • eISSN: 2508-5859 


\section{MATERIALS AND METHODS}

This study was conducted on patients who underwent HPB surgeries in Imam Khomeini Hospital Complex, Tehran University of Medical Sciences, Iran from 2018 to February 2020. Inclusion criteria were the adult patients who were candidates for HPB surgeries for malignant and nonmalignant diseases. Exclusion criteria were the patients who underwent simple laparoscopic cholecystectomy, and who were cigarette smokers or alcohol abusers. Patients who underwent surgery after from 2019 to February 2020 considered as the ERAS group $(n=47)$ in which ERAS was implemented postoperatively and the other patients $(n=43)$ were considered as control group in which conventional postoperative care was implemented. All patients were provided with the information about the possible harms and effects of the procedure and after obtaining an informed written consent included in in the study. The study protocol was approved by the Ethics Committees of our university and hospital.

Preoperative preparation was the same in all patients. No mechanical bowel preparation was performed preoperatively and clear oral fluid and solid food were allowed up to 4 and 6 hours before the start time of operation, respectively. Preoperative sedatives and anxiolytics were not given. A single dose of prophylactic antibiotic including Ampibactam $3 \mathrm{~g}$ was given intravenously to every patient 30-60 minutes before incision. All patients underwent epidural pain control. All HPB surgeries were performed by a same group of surgeons. Postoperative routine management in the both groups included enoxaparin $40 \mathrm{mg}$ daily subcutaneously to every patient up to 4 weeks after surgery, intravenous Ketorolac acid $30 \mathrm{mg}$ which was converted to oral nonsteroidal anti-inflammatory drugs when patient was orally allowed and continued till pain score was 4 or less according to the visual analogue scale and intravenous ranitidine $50 \mathrm{mg}$ every 8 hours. In patients with postoperative nausea and vomiting, metoclopramide $10 \mathrm{mg}$ was given each 8 hours intravenously and if not effective, ondansetron $4 \mathrm{mg}$ was given intravenously.

All patients were mobilized at postoperative day one. All were given incentive spirometry and abdominal belt. Head side of bed was kept elevated for 30 degrees. Wound dressing was changed 48 hours after surgery. Oxygen therapy with mask was started if oxygen saturation was less than $92 \%$.
In patients in the ERAS group, nasogastric tube was removed at 6 hours post operation and surgical diet was initiated. Tea, clear fluids, juices, and biscuits were given at postoperative day one and normal diet was started at postoperative day (POD) 2. Nasogastric tube, intraabdominal drains and Foley catheter were removed at POD 1. In the control group, diet was initiated as routinely based on surgeon opinion at POD 3 to 5; In patients without anastomosis diet was started at POD 1 and those with anastomosis, it was started at POD 4 or 5. In the control group, nasogastric tube was removed at POD 1 to 5 based on the surgeon opinion.

All patients filled in a questionnaire including demographic data, operative and postoperative data including type of surgical procedure, hospital stay, length of stay at intensive care unit and hospital, blood transfusions, nasogastric tube reinsertion, time for nasogastric tube removal, pain score at 6 hours after the operation according to the visual analogue scale (VAS) from zero for the minimum and ten for the maximum severity of pain, and postoperative complications were recorded prospectively.

Data was analyzed using SPSS software (IBM SPSS Statistics, USA). Descriptive and analytic results were reported using independent sample T-test, and chi-square test, respectively. A $p$-value less 0.05 was considered as statistically significant.

\section{RESULTS}

Ninety patients with the mean age of $47.3 \pm 13.3$ yrs/old (range=17-76) including 39 females were enrolled into the study. There was no significant difference between the demographic and preoperative comorbidities including diabetes mellitus, hypertension or coronary vascular diseases in the two groups. The mean age of the patients, number of females and patients with comorbid diseases in the ERAS vs. control group were $45 \pm 13$ vs. $49.8 \pm 13.3$ yrs/old ( $p=$ $0.081), 20$ vs. 19 patients $(p=1)$ and 11 vs. 7 patients $(p=$ $0.44)$, respectively. The type of the surgical procedures in each group is shown in Table 1 .

Postoperative data are shown in the Table 2. The mean duration of nasogastric tube and abdominal drain removal in the control group were $0.76 \pm 2$ days and $4.8 \pm 5$ days, respectively. As shown in the Table 2, except for the postoperative pain severity score there were no significant dif- 
Table 1. Type of the surgical procedures in each group

\begin{tabular}{lrcc}
\hline \multicolumn{1}{c}{ Type of surgical procedure } & $\begin{array}{c}\text { ERAS } \\
\text { group } \\
(\mathrm{n}=47)\end{array}$ & $\begin{array}{c}\text { Control } \\
\text { group } \\
(\mathrm{n}=43)\end{array}$ & $p$ \\
\hline Roux-en-Y hepaticojejunostomy & 10 & 2 & 0.029 \\
Right hepatectomy & 7 & 3 & 0.320 \\
Whipple procedure & 6 & 7 & 0.767 \\
Liver metastasectomy & 6 & 10 & 0.271 \\
Left hepatectomy & 5 & 3 & 0.716 \\
Hydatid cyst resection & 3 & 4 & 0.705 \\
Cholecystectomy & 2 & 3 & 0.667 \\
Partial hepatectomy & 2 & 6 & 0.145 \\
Choledochoduodenostomy & 1 & 0 & 1.000 \\
Distal pancreatectomy & 1 & 4 & 0.189 \\
Retroperitoneal mass resection & 2 & 0 & 0.495 \\
Pancreatic debridement & 1 & 0 & 1.000 \\
Total pancreatectomy & 1 & 0 & 1.000 \\
Hepatic cyst drainage & 0 & 1 & 0.478 \\
\hline
\end{tabular}

ERAS, enhanced recovery after surgery

ferences between the duration of ICU and hospital stay, units of transfused packed red blood cells, rate of nasogastric tube reinsertion, and postoperative complications between the two groups. Postoperative complications included hepatic failure, intraabdominal infection, pleural effusion, sepsis and wound infection. Bleeding, wound dehiscence, deep vein thrombosis and urinary tract infection were not seen in any patient.

Two patients in the ERAS group and one in the control group needed reoperation due to intraabdominal infection who were fully recovered and discharged with a good condition. One patient in each group died during hospitalization period due to myocardial infarction.

\section{DISCUSSION}

After releasing an evidence-based consensus recommendation for pancreatic surgery in 2012 there has been increasing number of clinical trials assessing the effectiveness of ERAS in the literature. ${ }^{10}$ However, there are still paucity of data and lack of larger multicentric trials to support ERAS in major hepatopancreaticobiliary surgeries. Also published protocols vary widely between different institutions.

After introduction of ERAS in colorectal surgery and following spread in other fields of surgery, there has been a trend toward using ERAS in major surgeries such as
Table 2. Postoperative data in the two groups

\begin{tabular}{lccr}
\hline \multicolumn{1}{c}{ Variable } & $\begin{array}{c}\text { ERAS } \\
\text { Group } \\
(\mathrm{n}=47)\end{array}$ & $\begin{array}{c}\text { Control } \\
\text { Group } \\
(\mathrm{n}=43)\end{array}$ & $p$ \\
\hline Duration of ICU stay, days & $1.7 \pm 2.2 *$ & $1.5 \pm .8$ & 0.735 \\
Duration hospital stay, days & $6.19 \pm 6$ & $6.44 \pm 5.2$ & 0.645 \\
PRBC transfusion, units & 6 & 4 & 0.883 \\
NG tube re-insertion & 6 & 2 & 0.270 \\
Postoperative complications & 12 & 9 & 0.628 \\
Pain severity, VAS score & $3.4 \pm 0.77$ & $4.47 \pm 0.88$ & $<0.001$ \\
\hline
\end{tabular}

*Mean \pm standard deviation; other data are presented number of patients

ICU, intensive care unit; PRBC, packed red blood cell; NG, nasogastric; VAS, visual analogue scale

HPB surgeries which indicated that ERAS could reduce hospital stay and postoperative complications. Liang et al. ${ }^{11}$ evaluated patients who underwent laparoscopic hepatectomies based on ERAS protocols and found that it could reduce postoperative hospital stay for nearly three days. A meta-analysis assessing 254 patients found that postoperative recovery time and length of hospital stay were significantly reduced in the ERAS group. ${ }^{12}$ However in our study, duration of hospital stay was not significantly different in the ERAS group compared to the control one which may be due to lower number of our studied patients or different types of surgical procedures.

In our study, patients were allowed to intake light meals up to 6 hours before the operation which probably decreases discomfort of patients in preoperative hours, besides might decrease catabolism and insulin resistance in some patients which is consistent with some other studies. ${ }^{13}$ This would help patients to pass a better operation and consequently less complicated postoperative period.

In our study, we removed abdominal drain in ERAS group faster than the control group which was not associated with an increased risk of intraabdominal complications like infection or biliary fistula compared to the control group. A study by Brauer et al., ${ }^{14}$ showed that drainage of the surgical site after hepatectomy does not improve diagnosis of biliary leaks, number of interventions, length of stay and 30-day readmissions.

However, a study by Kyoden et al. ${ }^{15}$ on 1269 patients who underwent hepatectomies found that prophylactic drainage was associated with reduction of probability of biliary fistulas and subphrenic pus collections. It seems that more 
powered studies are needed to come into a conclusion regarding routine drain insertion in HBP surgeries.

Patients in the ERAS group in our study experienced less pain compared to the control group. On the other hand, early oral feeding in the ERAS group was well tolerated and was not associated with higher rate of nausea or vomiting and postoperative complications Many studies have shown the benefits of early oral feeding, for example, Lee et al., ${ }^{16}$ indicated that early enteral diet could decrease hospital stay and faster return of gastrointestinal tract function in patients undergoing liver resection.

Finally, there was no significant difference regarding rates of postoperative complications, mortality and reoperation between the two groups. However, many trials have shown the beneficial effects of ERAS in reduction of morbidity and mortality rates compared to conventional postoperative care. A large meta-analysis including 3,694 patients was published recently assessing the impact of ERAS after pancreatic surgery which concluded that ERAS might be associated with a reduction of postoperative complications, especially abdominal infection, delayed gastric emptying and hospitalization days. However, there was no association between ERAS and rates of reoperation, readmission, postoperative pancreatic fistula or mortality. ${ }^{17}$

Limitations of our study were the small number of our patients and various types of HPB surgical procedures which might lead to some bias in interpreting results and generalizing them to other centers.

In conclusion, ERAS may be safe and effective in patients who undergo HPB surgery and may be associated with less severe postoperative pain.

\section{ACKNOWLEDGEMENTS}

We would like to thank the staff of our operation room who helped us in performing such major surgeries.

\section{CONFLICT OF INTEREST}

This was part of a thesis belonged to Dr. Zeeshan Hyder to obtain fellowship in hepatopancreaticobiliary surgery at Tehran University of Medical Sciences.

\section{ORCID}

Fakhar Nasir: https://orcid.org/0000-0002-0568-577X

Zeeshan Hyder: https://orcid.org/0000-0002-1081-1849

Amir Kasraianfard: https://orcid.org/0000-0002-3144-4451

Ali Sharifi: https://orcid.org/0000-0002-4179-202X

Abdolhamid Chavoshi Khamneh:

https://orcid.org/0000-0003-0675-3460

Seyed Yahya Zarghami:

https://orcid.org/0000-0003-2966-3679

Ali Jafarian: https://orcid.org/0000-0001-5375-8980

\section{AUTHOR CONTRIBUTIONS}

Conceptualization: FN, ZH, AJ. Data circulation: ZH, ACK. Formal Analysis: AK. Methodology: AS, SYZ. Project Administration: $\mathrm{ZH}$, AJ. Writing original draft: ZH. Writing - review \& editing: FN, ZH, AK.

\section{REFERENCES}

1. Kehlet H. Multimodal approach to control postoperative pathophysiology and rehabilitation. Br J Anaesth 1997;78:606-617.

2. Kehlet H, Wilmore DW. Evidence-based surgical care and the evolution of fast-track surgery. Ann Surg 2008;248:189-198.

3. Azhar RA, Bochner B, Catto J, Goh AC, Kelly J, Patel HD, et al. Enhanced recovery after urological surgery: a contemporary systematic review of outcomes, key elements, and research needs. Eur Urol 2016;70:176-187.

4. Gustafsson UO, Scott MJ, Schwenk W, Demartines N, Roulin D, Francis N, et al. Guidelines for perioperative care in elective colonic surgery: Enhanced Recovery After Surgery (ERAS ${ }^{\mathbb{R}}$ ) Society recommendations. Clin Nutr 2012;31:783-800.

5. Pisarska M, Małczak P, Major P, Wysocki M, Budzyński A, Pędziwiatr M. Enhanced recovery after surgery protocol in oesophageal cancer surgery: systematic review and meta-analysis. PLoS One 2017;12:e174382.

6. Kaibori M, Matsui K, Ishizaki M, Iida H, Yoshii K, Asano H, et al. Effects of implementing an "enhanced recovery after surgery" program on patients undergoing resection of hepatocellular carcinoma. Surg Today 2017;47:42-51.

7. Savikko J, Ilmakunnas M, Mäkisalo H, Nordin A, Isoniemi H. Enhanced recovery protocol after liver resection. Br J Surg 2015; 102:1526-1532.

8. Melloul E, Hübner M, Scott M, Snowden C, Prentis J, Dejong $\mathrm{CH}$, et al. Guidelines for perioperative care for liver surgery: Enhanced Recovery After Surgery (ERAS) Society recommendations. World J Surg 2016;40:2425-2440.

9. Bai X, Zhang Q, Gao S, Lou J, Li G, Zhang Y, et al. Duct-tomucosa vs invagination for pancreaticojejunostomy after pancreaticoduodenectomy: a prospective, randomized controlled trial from a single surgeon. J Am Coll Surg 2016;222:10-18.

10. Lassen K, Coolsen MM, Slim K, Carli F, de Aguilar-Nascimento JE, Schäfer M, et al. Guidelines for perioperative care for pancreaticoduodenectomy: Enhanced Recovery After Surgery (ERAS ${ }^{\mathbb{R}}$ ) 
Society recommendations. Clin Nutr 2012;31:817-830.

11. Liang X, Ying H, Wang H, Xu H, Yu H, Cai L, et al. Enhanced recovery program versus traditional care in laparoscopic hepatectomy. Medicine (Baltimore) 2016;95:e2835.

12. Li L, Chen J, Liu Z, Li Q, Shi Y. Enhanced recovery program versus traditional care after hepatectomy: a meta-analysis. Medicine (Baltimore) 2017;96:e8052.

13. Francisco SC, Batista ST, Pena Gd. Fasting in elective surgical patients: comparison among the time prescribed, performed and recommended on perioperative care protocols. Arq Bras Cir Dig 2015;28:250-254.

14. Brauer DG, Nywening TM, Jaques DP, Doyle MB, Chapman WC, Fields RC, et al. Operative site drainage after hepatectomy: a propensity score matched analysis using the American College of Surgeons NSQIP Targeted Hepatectomy Database. J Am Coll Surg 2016;223:774-783.e2.

15. Kyoden Y, Imamura H, Sano K, Beck Y, Sugawara Y, Kokudo $\mathrm{N}$, et al. Value of prophylactic abdominal drainage in 1269 consecutive cases of elective liver resection. J Hepatobiliary Pancreat Sci 2010;17:186-192.

16. Lee J, Kwon CH, Kim JM, Shin M, Joh JW. Effect of early enteral nutrition after hepatectomy in hepatocellular carcinoma patients. Korean J Hepatobiliary Pancreat Surg 2012;16:129-133.

17. Ji HB, Zhu WT, Wei Q, Wang XX, Wang HB, Chen QP. Impact of enhanced recovery after surgery programs on pancreatic surgery: a meta-analysis. World J Gastroenterol 2018;24:1666-1678. 